\title{
Work-related musculoskeletal discomfort and injuries in Australian optometrists
}

\author{
Jennifer Long ${ }^{\mathrm{a},{ }^{*}}$, Robin Burgess-Limerick ${ }^{\mathrm{b}}$ and Fiona Stapleton ${ }^{\mathrm{a}}$ \\ ${ }^{a}$ School of Optometry and Vision Science, University of New South Wales, Sydney NSW 2052, Australia \\ ${ }^{\mathrm{b}}$ Minerals Health and Safety Centre, Sustainable Minerals Institute, University of Queensland, Brisbane QLD \\ 4072, Australia
}

\begin{abstract}
Work-related musculoskeletal discomfort and injuries are reported by optometrists. The purpose of this paper is to describe the process used to investigate work-related discomfort in Australian optometrists. A multistage project was conducted which included questionnaires, interviews and onsite observations. Participants, with and without self-reported discomfort, included clinical optometrists, optometry students and clinical teachers. The various stages of the study developed over the course of the project, primarily in response to results collected at various stages. A multistage approach proved valuable for confirming results and testing hypotheses, and for investigating different groups of workers (clinicians, teachers and students). General guidelines to reduce the risk of work-related discomfort in optometrists can be developed from this project. Specific recommendations and teaching curricula have been identified in this project as areas of future research and development.
\end{abstract}

Keywords: education, guidelines, interview, observation, questionnaire

\section{Introduction}

Optometry is a primary healthcare profession with 4429 registered practitioners in Australia [1]. The role of an optometrist is to provide eye and vision care which includes determining an optical correction (e.g. spectacles, contact lenses), detecting / diagnosing/ managing eye diseases and assisting with the rehabilitation of eye conditions [2]. Optometrists often work with small teams of people which may include a practice manager, receptionist and optical dispenser. Larger practices may have multiple optometrists working from the same premises and ophthalmic assistants to administer automated testing procedures. Optometrists may work full-time in one practice, part-time between multiple practices or on a short-term (locum) basis. Workloads vary between practices but the typical duration of an eye examination is 20-30 minutes. Therefore, a busy optometrist may examine up to 25 patients per day.

Optometrists perform several different clinical procedures during a consultation. Although each individual procedure may take less than five minutes to complete, many procedures require sustained postures, awkward postures and fine motor control of the fingers. Subsequently, there have been reports of physical discomfort within the ophthalmic profession $[5,8,7]$ and anecdotal reports of optometrists who experience work-related discomfort on a day-to-day basis, who have modified their work hours or work tasks and who have even left the profession.

The initial purpose of this investigation was to substantiate reports of work-related discomfort in Australian optometrists. This was achieved by issuing a questionnaire to obtain an estimate of the type, severity and independent risk factors for discomfort. However, the questionnaire results raised issues which warranted further investigation, leading to an expansion of the project scope.

The purpose of this paper is to:

- Describe the process used to investigate work-related musculoskeletal discomfort and injuries in Australian optometrists

- Discuss some of the issues associated with developing guidelines for clinical practice to reduce work-related discomfort and injuries.

${ }^{*}$ Corresponding author. Telephone +61 247823472 E-mail: j.long@unsw.edu.au 


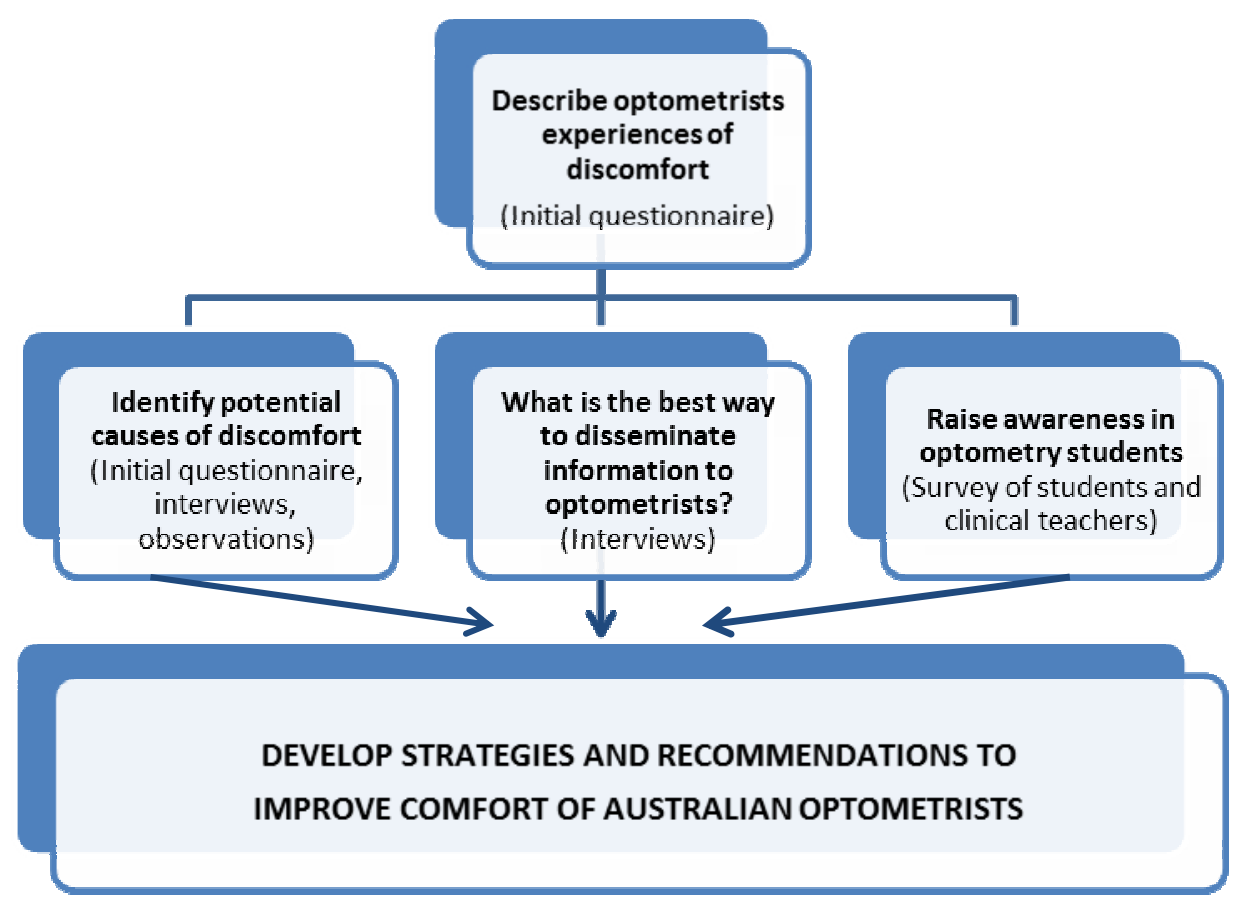

Figure 1. Project overview

\section{Methods}

This multi-stage study included:

- An initial questionnaire sent by email to members of the Optometrists Association of Australia (OAA) in August 2008, based on the Nordic Musculoskeletal Questionnaires [10] and job factors identified by Bork et al [4]. A more detailed description of the methodology is reported elsewhere [14]

- $\quad 30$ minute telephone or face-to-face interviews with optometrists. These were semi-structured and included questions relating to demographics, job satisfaction, description of discomfort and ergonomics. A more detailed description of the methodology is reported elsewhere $[12,11]$

- Onsite observations of 10 optometrists with video recording of the participant conducting an eye examination. Analysis of the video recordings were conducted with RULA (Rapid Upper Limb Assessment)[16] and ManTRA (Manual Tasks Risk Assessment) [6]

- A survey of optometry clinical teachers at three Australian teaching institutions (University of New South Wales, University of Queensland, Australian College of Optometry) and at one
New Zealand teaching institution (University of Auckland) to determine what type of teaching is given to optometry students to reduce their risk of work-related discomfort.

- A survey of optometry students enrolled in the Bachelor of Optometry and Bachelor of Vision Science program at UNSW. The purpose was to determine if optometry students experience discomfort when performing clinical procedures and their preferences for receiving information how to reduce their risk of discomfort. A description of the methodology used in the surveys of clinical teachers and optometry students is reported elsewhere [13].

An overview of the approach is shown in figure 1 . All stages of investigation were approved by the Human Research Ethics Advisory Panel of the University of New South Wales.

\section{Results}

\subsection{Questionnaire}

The project was developed by the first author following anecdotal reports of work-related discomfort by optometrists. The initial questionnaire was distri- 
buted to determine the type, severity and independent risk factors for discomfort in Australian optometrists.

There were 416 optometrists $(n=233$ females, $56 \%$ ) who participated in the questionnaire, which represents approximately $25 \%$ of optometrists with active email addresses when the questionnaire was issued [14]. Of these respondents, $82 \%$ reported work-related discomfort, most commonly in the neck, shoulder and lower back. The results showed that the risk of reporting discomfort increased for female gender (OR 6.6 CI=2.2-19.9) and those who conduct a greater number of eye examinations per day (OR 5.1 CI 2.1-12.7) and the risk of experiencing severe discomfort (i.e. discomfort present for greater than 30 days) increased for those who perform repetitive tasks (OR 1.9 $\mathrm{CI}=1.2-3.1)$ and who continue to work while injured (OR 2.9, CI=1.6-5.2).

While this confirmed initial suspicions that workrelated discomfort exists in the optometry profession, it also raised many more questions. For example, population attributable risk analysis showed that eliminating repetitive tasks and ceasing to work while injured would reduce the disease load for severe discomfort by $28 \%$ - but this means that $72 \%$ of the risk is unaccounted for.

\subsection{Interviews}

Telephone interviews were conducted with 60 optometrists ( $\mathrm{n}=47$ with self-reported discomfort) to further explore reasons for work-related discomfort and strategies used to reduce discomfort. All participants were asked about work satisfaction and their ability to control their working environment.

At this stage of project development, there were enquiries from members of the profession for practical advice to reduce work-related discomfort. It became clear that guidelines or recommendations would be a useful outcome of the project. With this in mind, the interview schedule of questions was expanded to include questions about ergonomics and how the participant would prefer to access information aimed at reducing work-related physical discomfort.

There were six participants who reported that they have modified their work hours as a result of workrelated discomfort ( $n=2$ medically retired, $n=4$ work reduced hours) and some participants reported spending several hundred Australian dollars per month simply to keep their pain under control. Two ophthalmic tasks were commonly described as contributing to discomfort (refraction and slit lamp). This sug- gested that another stage of investigation - onsite observation and postural analysis of clinical tasks may provide useful information to assist with the development of guidelines and recommendations for the profession.

When asked their opinion on accessing ergonomics information to reduce the risk of work-related discomfort, participants reported that they would access information [12] and were open to implementing a participatory ergonomics approach in their practices [11]. There were no significant relationships between preference for obtaining information and personal experience of work-related physical discomfort. Although this cohort were generally interested in ergonomics, many felt that it is too late to educate optometrists already in practice about workrelated discomfort as habits have already been formed and equipment purchased and installed. This prompted the development of an investigation of optometry students and clinical teachers.

\subsection{Onsite observations}

There were 10 optometrists who participated in the onsite observations ( $\mathrm{n}=8$ with self-reported discomfort). RULA analysis appears to be a more sensitive indicator of the risk of discomfort associated with individual ophthalmic tasks. This is consistent with the questionnaire results (section 3.1) showing that optometrists are more likely to report upper body discomfort.

These observations can be used as case studies illustrating examples of good and bad practice and techniques when guidelines for optometrists are developed.

\subsection{Survey of optometry students and optometry clinical teachers}

The feasibility of raising awareness of workrelated discomfort in optometry students was investigated using two surveys. There were 64 optometry students ( $48 \%$ response rate, $n=45$ females) and 46 clinical teachers $(30 \%$ response rate) who participated in this stage of the project [13].

Of the 64 optometry students who participated, $77 \%$ reported physical discomfort in the previous 12 months while performing clinical procedures, most commonly in the lower back, neck, shoulder and upper arm. This indicates that work-related discomfort can occur early in an optometrist's professional career. Although clinical teachers do not receive formal 
instruction to assist students reduce their risk of work-related discomfort, the majority of respondents reported that they do provide informal instruction, for example, correct a student when they are observed using a poor posture.

\section{Discussion}

Publications exist in the ophthalmic literature describing work-related discomfort and possible ways to reduce discomfort. These publications are primarily based on the author's experience of discomfort or their observations in the consultation room $[9,15,3]$. To our knowledge there has not been a systematic analysis of the extent of work-related discomfort within optometry, nor analyses of the risk factors, strategies adopted by clinicians, costs or consequences of injury. This project, which consisted of multiple stages of investigation, established that work-related discomfort exists in optometry and has attempted to systematically investigate this with a view to developing evidence-based recommendations and guidelines for clinical practice.

The multistage approach allowed flexibility in the development of ideas and the methodology over the course of the project. For example, comments by some of the interviewees that students should be given instruction led to the investigation of clinical teachers and students. The flexible approach also averted conducting large numbers of onsite observations once it became clear that it was impractical to make observations of all possible working environments and equipment.

The multistage approach was also important for substantiating and confirming information that was gathered, an important methodology within qualitative research [17]. For example, the initial questionnaire asked participants if they have been able to modify their work or workspace to decrease their discomfort. Although this was valuable for understanding the diversity of methods used by optometrists in the consultation room, the interviews gave the opportunity for participants to elaborate on their responses.

Except for the use of the Nordic Musculoskeletal Questionnaire, the methodology was essentially exploratory. This approach was necessary since there is little published in the scientific literature on workrelated injuries in optometrists. There was also response bias in that optometrists who were interested in participating or who have experienced work- related discomfort elected to participate and these optometrists may not be representative of all optometrists in clinical practice. On the other hand, this project represents the first step in understanding the issues of discomfort in this professional group and is useful for the development of more formal investigation tools. For example, open ended questions were asked in the interviews to better understand how optometrists prefer to gain information about ergonomics to reduce the risk of work-related discomfort. This will assist in the construction of a participatory ergonomics program which has widespread acceptance by optometrists.

The principle difficulty encountered in this multistage project was becoming conversant with a wide range of methodologies and bodies of knowledge e.g. education, musculoskeletal assessment tools, psychosocial factors, professional development. A1though a challenge, this holistic approach is a cornerstone of ergonomics and reflects the multifaceted nature of work-related discomfort.

\subsection{To the future}

The initial goal to develop guidelines has proved more elusive than first envisioned. This is due to the diversity of equipment, practice styles, work practices, room arrangements and individual body dimensions. While it is possible to develop some generic guidelines for clinical optometrists, specific advice may be better given on an individual as-needs basis.

This project has identified several potential areas for future research. For example:

- Questionnaire and interview participants identified equipment design and room design as contributing factors to their discomfort. There is scope for further investigation into these issues with a view to making specific recommendations about ophthalmic equipment and consultation room design.

- Participatory ergonomics programs may assist optometrists reduce work-related discomfort. The results of this project provide guidance for strategies which may be attractive to optometrists. However, the actual participatory programs need to be developed and tested for effectiveness

- RULA appears to be a more sensitive indicator for risk of discomfort compared to ManTRA for individual ophthalmic tasks. However, to assess the total risk of discomfort in optometrists it may be necessary to undertake more detailed biomechanical analysis, particularly to evaluate and 
document improvements in the use of alternative equipment.

- It is beyond the scope of this current study to develop and test appropriate curricula for optometry students. However, the results of this project indicate that a three-way strategy may be required to reduce the risk of discomfort in optometry students: educate the students, educate the educators and maximize student exposure to good practice.

The best outcome for reducing work-related discomfort in optometrists is to involve the whole profession - clinicians, academics, students, industry - in the problem solving process. By doing this, a holistic rather than a fragmented solution will be achieved.

\section{References}

[1] 4,429 registered optoms in Aus, mivision, Toma Publishing, Australia, June (2011), pp. 5

[2] About Us, World Council of Optometry. http://www.worldoptometry.org/en/aboutwco/index.cfm Accessed 12 $2^{\text {th }}$ May 2011

[3] Working safely in optometry, Optometry News (OAA Victoria) (2007)

[4] B. Bork, T. Cook, J. Resencrance, K. Engelhardt, M. Thomason, I. Wauford and R. Worley, Work-Related Musculoskeletal Disorders Among Physical Therapists, Physical Therapy, 76 (1996), pp. 827-835.

[5] A. Bruce and K. Snibson, A pain in the neck for optometrists, In focus, 6 (2007), pp. 4--5.

[6] R. Burgess-Limerick, Procedure for Managing Injury Risks Associated with Manual Tasks, BurgessLimerick \& Associates, 2008.

[7] A. Chiang, P. Baker, E. Milder and S. Garg, Ergonomics for the Retina Specialist, Retina Today, July/August (2010), pp. 25-26.
[8] K. Dhimitri, G. McGwin, S. McNeal, P. Morse, M. Patterson and J. Marx, Symptoms of Musculoskeletal Disorders in Ophthalmologists, American Journal of Ophthalmology, 139 (2005), pp. 179-181

[9] R. Hutchins and J. Schneebeck, Doctor, Ergonomic Thyself, Journal of Behavioral Optometry, 15 (2004), pp. 87-89.

[10] I. Kuorinka, B. Jonsson, A. Kilbom, H. Vinterberg, F. Biering-Sorensen, G. Andersson and K. Jorgensen, Standardised Nordic questionnaires for the analysis of musculoskeletal symptoms, Applied Ergonomics, 18 (1987), pp. 233-237.

[11] J. Long, R. Burgess-Limerick and F. Stapleton, Acceptance of participatory ergonomics in a healthcare setting, in R. Burgess-Limerick, ed., Safer and more productive workplaces: Proceedings of the 46th Annual Conference of the Human Factors and Ergonomics Society of Australia, HFESA Inc., Sunshine Coast, Queensland, 2010, pp. 43-52.

[12] J. Long, R. Burgess-Limerick and F. Stapleton, Toward a comfortable profession - disseminating ergonomics information to Australian optometrists, Ergonomics Australia, (2011) 7:1

[13] J. Long, Y. Ko, C. Lau, R. Burgess-Limerick and F. Stapleton, Developing strategies for reducing workrelated discomfort in optometry students, Ergonomics Australia, in press (2011).

[14] J. Long, T. Naduvilath, H. Ling, A. Li, W. Ng, W. Yip and F. Stapleton, Risk factors for physical discomfort in Australian optometrists, Optometry and Vision Science, 88 (2011), pp. 317-326.

[15] J. Marx, F. Wertz and K. Dhimitri, Work-related musculoskeletal disorders in ophthalmologists, Techniques in Ophthalmology, 3 (2005), pp. 54-61.

[16] L. McAtamney and E. Corlett, RULA: a survey method for the investigation of work-related upper limb disorders, Applied Ergonomics, 24 (1993), pp. 91-99.

[17] J. Morse, M. Barrett, M. Mayan, K. Olen and J. Spiers, Verification strategies for establishing reliability and validity in qualitative research, International Journal of Qualitative Methods, 1 (2002), pp. 13-22. 